\title{
On RiEmannian GeOMETRIES of Visual SpaCE
}

\author{
Jacek Turski* \\ Department of Mathematics and Statistics \\ University of Houston-Downtown \\ Houston, TX 77002 \\ turskij@gmail.com
}

January 11,2022

\begin{abstract}
This is a study of the phenomenal geometries constructed in the Riemannian geometry framework from simulated iso-disparity conics in the horizontal visual plane of the binocular system with the asymmetric eyes (AEs). For the eyes' resting vergence posture, which depends on parameters of the $\mathrm{AE}$, the iso-disparity conics are frontal straight lines in physical space. For all other fixations, the simulated iso-disparity conics consist of families of the ellipses or hyperbolas depending on both the AE's parameters and the bifoveal fixation. However, the iso-disparity conic's arcs are perceived in the gaze direction as the frontal lines. Thus, geometries of physical and visual spaces are different. An assumption underlying the relevant architecture of the human visual system is combined with results from simulated iso-disparity straight lines, giving the relative depth as a function of the AE's parameters and the distance. This establishes the metric tensor in binocular space of fixations for the eyes' resting vergence posture. The resulting geodesics in the gaze direction, give the distance to the horizon and zero curvature. For all other fixations, only the sign of the curvature may be inferred from the global behavior of the simulated iso-disparity conics.
\end{abstract}

Keywords Binocular correspondence, asymmetric model eye, iso-disparity conics, visual space, Riemannian geometry, metric tensor, geodesics, curvature

\section{Introduction}

When a small retinal area is stimulated in one of the two eyes, there is a unique retinal area in the other eye such that when it is stimulated, a single perceptual phenomenon localized in our phenomenal visual space corresponds to both stimulated areas. These two retinal areas are referred to as corresponding retinal elements and we consider them as having zero disparity. For a given fixation, the horopter is defined as the locus of points in space such that each point on the horopter projects to a pair of corresponding retinal elements. Thus, it is the spatial curve of zero-disparity points.

A small object that is located away from the horopter has nonzero disparity and, in general, is perceived as two objects. However, if the object is located close to the horopter, the brain fuses its disparate images into a single percept and the decussation at the optic chiasm allows images from the two retinae to be compared. The extracted horizontal disparity is used by the brain to create our sense of depth relative to the horopter. Even the objects located farther from the horopter, which lead to double vision, convey the sense of depth [19].

More essentially, the difference in horizontal disparity between two spatial points is used by the brain to sense the relative depth and, hence, to our perception of objects' shapes and their relative locations in 3D space, that is, stereopsis and visual space geometry. Because the eyes' lateral separation, stereopsis is mainly supported by the horizontal retinal correspondence and the longitudinal horopter.

\footnotetext{
${ }^{*}$ Professor Emeritus
} 
The outcome of the above outlined physiological processes is the beholder's three-dimensional visual experience combined with other perceptual modalities and transcended by higher cognitive processes. This article concerns our phenomenal visual space geometric relations before the involvement of psychological processes.

Though the study of phenomenal visual space has had a long history, the geometric relationship between phenomenal visual space and the stimuli-containing physical space remains unresolved. The main reason is that the spatial relations of phenomenal space such as depth and size are, in general, task-dependent and influenced by both contextual factors and our expectations. However, the binocular disparity provides the most precise depth cue that is sufficient for stereopsis with no explicit recognition of a scene's geometric forms such that the resulting perception is generally unaffected by other factors.

Here, I investigate the disparity-based phenomenal geometry in the binocular system with the asymmetric eyes (AEs) in the framework of the bicentric perspective projections [26]. The AE model is comprised of the fovea's displacement from the posterior pole by angle $\alpha=5.2^{\circ}$ and the crystalline lens' tilt by angle $\beta$. Angle $\alpha$ is relatively stable in the human population and angle $\beta$ varies between $-0.4^{\circ}$ and $4.7^{\circ}$, cf. the discussion in [25]. In the human eye, the fovea's displacement from the posterior pole and the cornea's asphericity contribute to optical aberrations that the lens' tilt then partially compensates for [1].

The horopteric conics simulated in [26] resemble the empirical horopters and are extended in this study to the families of iso-disparity conics for a stationary, upright head with the eyes converging on the points of the horizontal binocular field of fixations. For the eyes in the resting vergence posture, that is, when the eyes fixate at the abathic distance, the iso-disparity curves are straight frontal lines. For other fixations, the iso-disparity curves consist of either a family of ellipses or hyperbolas, depending on the AE's asymmetry parameters and the location of the fixation point.

However, the iso-disparity curves are always perceived in the gaze direction as the frontal lines. Therefore, the geometries of physical space and phenomenal visual space are different and the global aspects of this difference are studied here in the framework of Riemannian geometry.

\section{Retinal Correspondence}

The correspondence of retinal elements as described in the first section, forms the basis of the nonius paradigm for a direct determination of the corresponding retinal elements [20, 22]. All other methods, as reviewed in [27], are indirect and less reliable.

However, retinal correspondence is not readily available in nonius measurements of longitudinal horopter [27]. More importantly, it cannot be obtained from geometrical methods because of the beholder eye's asymmetry: the corresponding points are compressed in the temporal retinae relative to those in the nasal retinae [23]. This situation changed when the geometric theory of the binocular system with AEs was developed in [26] that fully specified the retinal correspondence in terms of AE's asymmetry parameters. It was verified in simulations of the horopteric conics starting from the resting vergence eyes' posture shown in Figure 1.

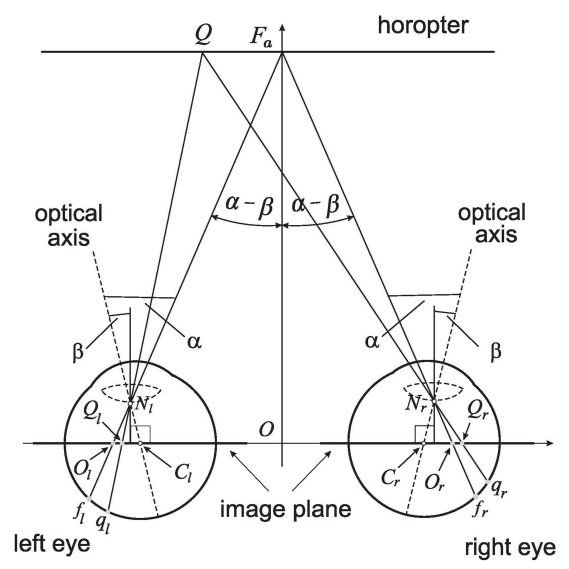

Figure 1: Retinal correspondence. The linear horopter passing through the fixation $F_{a}$ at the abathic distance $d_{a}=\left|O F_{a}\right|$. The fixation point $F_{a}$ projects to the foveae $f_{r}$ and $f_{l}$ and the point $\mathrm{Q}$ on the horopter projects to the retinal corresponding points $q_{r}$ and $q_{l}$ and to points $Q_{r}$ and $Q_{l}$ in the image plane. The subtense $\sigma_{a}$ at $F_{a}$ is given by $2(\alpha-\beta)[26]$. 
I briefly review the retinal correspondence in the binocular system with AEs established in [26] because in Section 4 it is used to formulate the extension of the horopteric conics (zero-disparity conics) to the iso-disparity conics.

The distribution of the retinal corresponding points $q_{r}, q_{l}$ is asymmetrical with respect to the foveae $f_{r}$ and and $f_{l}$ such that $\left|q_{r} f_{r}\right| \neq\left|q_{l} f_{l}\right|$, see Figure 1] It is demonstrated in [26], that the distribution of these corresponding points projected into the image planes of each AE, $Q_{r}, Q_{l}$, satisfy $\left|Q_{r} O_{r}\right|=\left|Q_{l} O_{l}\right|$ where $O_{r}$ and $O_{l}$ are projected foveae $f_{r}$ and $f_{l}$, respectively. It is proved in [26] that this symmetry on the image plane is preserved for all other fixations. Thus, unknown (asymmetric) retinal correspondence can be entirely formulated on the image plane of the $\mathrm{AE}$ in terms of the asymmetry parameters.

The abathic distance $d_{a}=\left|O F_{a}\right|$ to the fixation point $F_{a}$ in the eyes' resting vergence posture was obtained in [26] as follows:

$$
d_{a}=\frac{a \cos (\alpha-\beta)+0.6 \sin \alpha}{\sin (\alpha-\beta)},
$$

where $2 a=6.5 \mathrm{~cm}$ is the ocular separation and $0.6 \mathrm{~cm}$ is the distance of the nodal point from the eyeball's rotation center.

I close this section by explaining why the eyes fixating at the abathic distance are referred to as the eyes' resting vergence posture, or later as the resting eyes for short. The image plane in the AE model is parallel to the lens's equatorial plane and is passing through the eye's rotation center. Figure 1 shows the eyes' posture in which the image planes are coplanar and the horopter is frontal straight line passing through the fixation point at the abathic distance. This distance numerically corresponds to the eyes' resting vergence posture [13]. Because the eye muscles' natural tonus resting position serves as a zero-reference level for convergence effort [7], this eye posture is identified in [26] with the eyes' binocular primary position.

\section{Binocular Field of Fixations}

For an observer with a stationary, upright head and binocular fixations in the horizontal plane, the coordinate system consists of the $y$-axis passing through the eyes' rotation centers and the head $z$-axis formed by the intersection of the midsagittal plane with the horizontal visual plane, see Figure 2

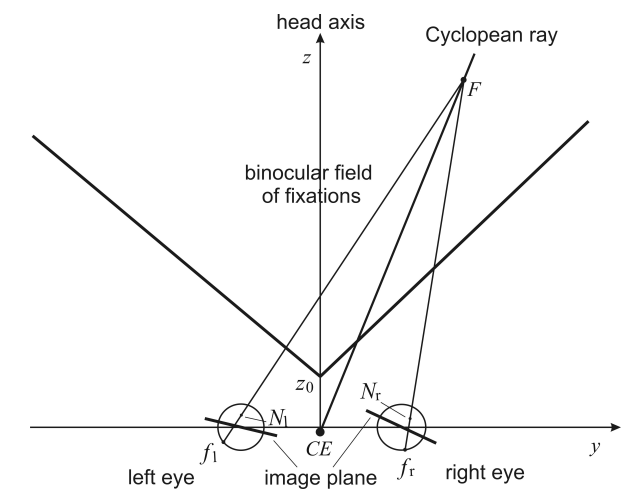

Figure 2: The binocular field of fixations in the horizontal visual plane for the binocular system with the AEs.

The binocular field of fixations is limited by the head/eyes anatomy and physiology: the protruded nose and the fixations neurally restricted to the azimuthal range $\pm 45^{\circ}[10]$. Further, the geometry of the eyes in symmetric convergence shown in Figure 1. demonstrate that the binocular system possesses bilateral symmetry-the symmetry with respect to the head's midsagittal plane. Therefore, the binocular field of fixation, denoted by $B$, is assumed symmetric about the z-axis. Thus, $B$ is a convex set shown in Figure 2 where the point $\left(0, z_{0}\right)$ denotes the closest point on which the eyes can converge. For fixation point, the monocular perception from outside of $B$ is still available. In fact, the perceived Cyclopean direction, specified by the Cyclopean ray in (2), does not depends on whether the stimulus reaches the retinal element in one eye or its corresponding element in the other eye alone or whether it reaches both the corresponding element simultaneously [27]. However, stereopsis is not available in the region outside of $B$.

For a fixation point, the local chart for the binocular visual space is the whole set $B$. For every fixation $(y, z) \in B, \mathrm{I}$ want to find the corresponding scalar product on the tangent plane, $R^{2}$, with its origin at $(y, z)$, using the biologically 
bioRxiv preprint doi: https://doi.org/10.1101/2021.10.25.465820; this version posted January 11,2022 . The copyright holder for this preprint (which was not certified by peer review) is the author/funder. All rights reserved. No reuse allowed without permission.

A PREPRINT - JANUARY 11, 2022

motivated geometric theory of human binocular perception developed in [26]. These families of scalar products will define the Riemannian geometries of visual space, each dependent on both the AEs parameters and the point of binocular fixation.

\section{Simulation of Iso-Disparity Curves}

The iso-disparity conics are first specified on the image planes of the AEs when the eyes are in the resting vergence posture shown in Figure 1. Referring to this figure, recall that points $Q_{r}$ and $Q_{l}$ on the same side and of the same distance from the centers $O_{r}$ and $O_{l}$ are the corresponding points covering the corresponding retinal elements $q_{r}$ and $q_{l}$. The corresponding points back-projected through the nodal points give the spatial point $Q$ that is on the horopter line.
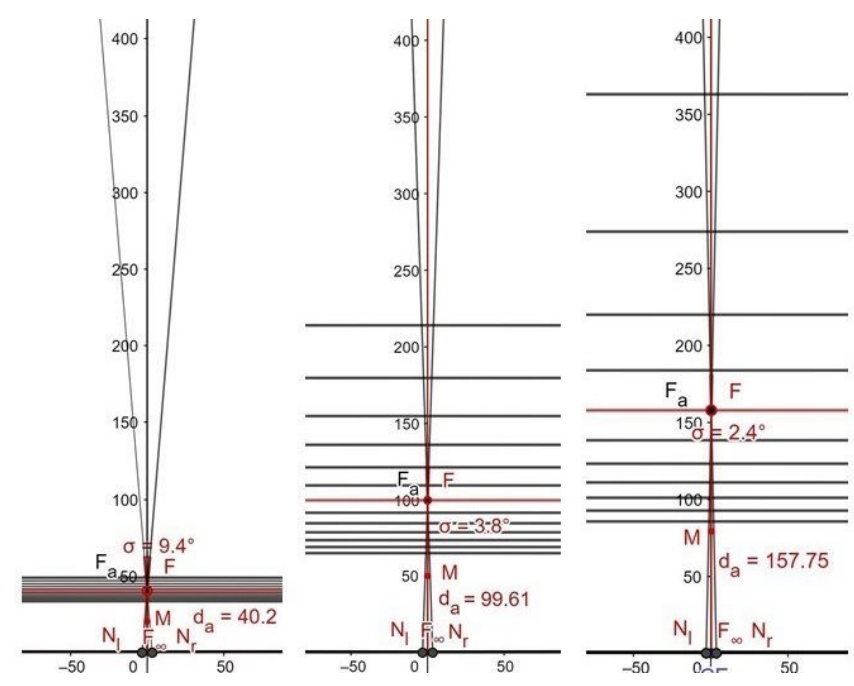

Figure 3: The iso-disparity lines for the eyes' resting vergence fixations with the abathic distance given in (1): on the left, the abathic distance $d_{a}=40.2 \mathrm{~cm}$ for $\beta=0.5^{\circ}$, in the middle, the abathic distance $d_{a}=99.61 \mathrm{~cm}$ for $\beta=3.3^{\circ}$, and on the left, the abathic distance $d_{a}=157,75 \mathrm{~cm}$ for $\beta=4^{\circ}$. The angles at $F$ are $\sigma=2(\alpha-\beta)$, where $\alpha=5.2^{\circ}$. The horopters are shown in horizontal red lines.
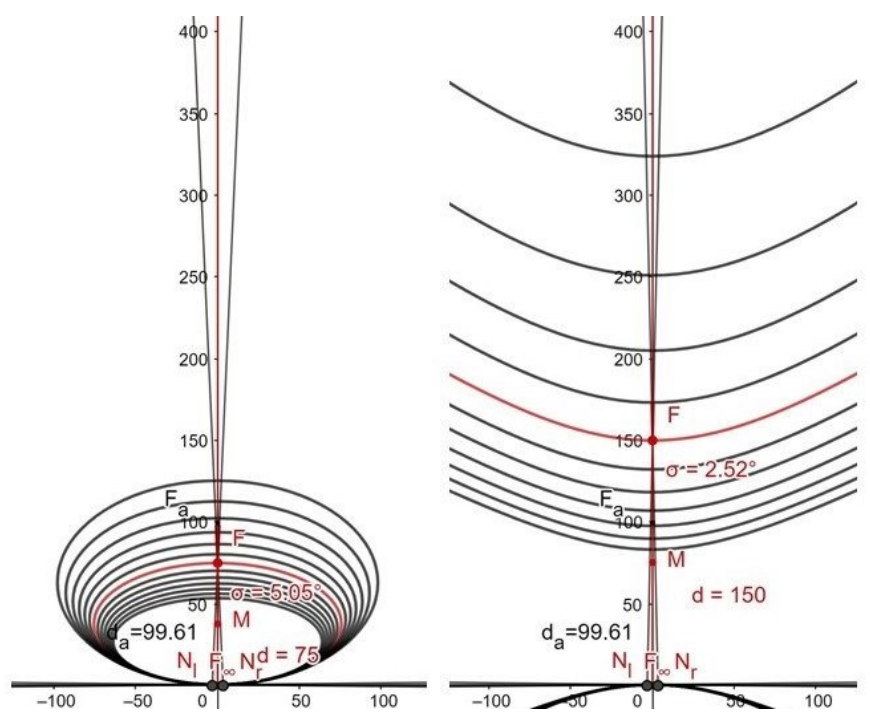

Figure 4: The iso-disparity conics for the binocular system of the abathic distance $d_{a}=99.61 \mathrm{~cm}$ for two symmetric fixations at the indicated distances $d$ : ellipses on the left and hyperbolas on the right. The conics in red lines are horopters. 
bioRxiv preprint doi: https://doi.org/10.1101/2021.10.25.465820; this version posted January 11, 2022. The copyright holder for this preprint (which was not certified by peer review) is the author/funder. All rights reserved. No reuse allowed without permission.

A PREPRINT - JANUARY 11, 2022

The iso-disparity lines are constructed as follows. The points $Q_{r}+\delta_{0}$ and $Q_{l}$ on the $y$-coordinate line, when backprojected, gives the crossed point on the $\delta_{0}$-disparity line. Similarly, the points $Q_{r}-\delta_{0}$ and $Q_{l}$, when back-projected, give the uncrossed point on the $-\delta_{0}$-disparity line.
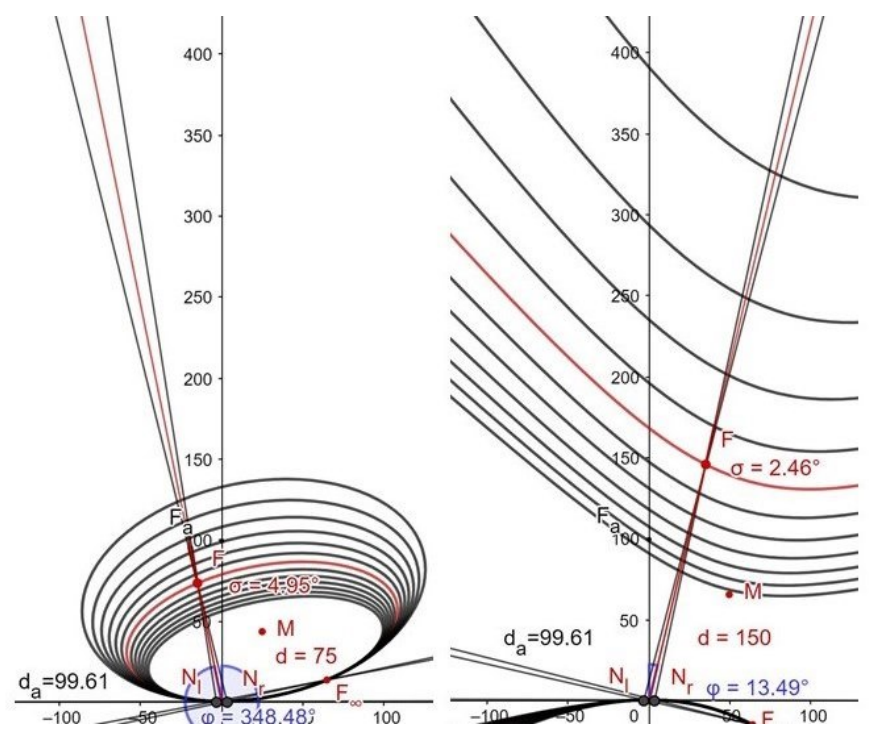

Figure 5: The iso-disparity conics for two asymmetric fixations: ellipses on the left and hyperbolas on the right. The conics in red lines are horopters. The straight line passing through the fixation $F$ is the Cyclopean ray. Its direction specifies the orientation of the conics. The indicated distances $d$ are measured along the Cyclopean ray.

The simulations of the iso-disparity conics of a constant disparity difference that are carried out in GeoGebra are shown in Figure 3, Figure 4 and Figure 5 for the same relative disparity $\delta_{0}=0.00355 \mathrm{~cm}$.

Figure 4 and Figure 5 show how the iso-disparity lines for the case of $d_{a}=99.61 \mathrm{~cm}$ in Figure 3 are transformed into ellipses and hyperbolas for symmetrically (Figure 4) and asymmetrically (Figure 5) convergent eyes in the binocular field. The fixation points are of the distance $75 \mathrm{~cm}$ and $150 \mathrm{~cm}$ along the Cyclopean ray for the ellipses and hyperbolas, respectively. The direction of the Cyclopean ray specifies the orientation of the horopteric conics [26] and, therefore, the orientations of the iso-disparity conics for a given fixation.

\section{Relative Depth for Resting Eyes}

From the simulations shown in Figure 3 for the disparity step of $\delta_{0}=0.00355 \mathrm{~cm}$, I calculate the distance $\Delta z_{i}$ and midpoint $z_{i}$ between the consecutive iso-disparity lines to approximate how the depth of iso-disparity lines changes with distance to the head in the gaze's direction. The points $\left(z_{i}, \Delta z_{i}\right)$ for different abathic distance eyes fixations lie on the graph of a single quadratic function

$$
\Delta z\left(z, \delta_{0}\right)=0.000892 z^{2}
$$

shown in Figure 6

To investigate the iso-disparity lines distribution dependence on disparity value, I compare simulated iso-disparity lines for disparity difference $\delta_{0}=0.00355 \mathrm{~cm}$ with lines simulated for disparity $\delta=(1 / 5) \delta_{0}=0.00071 \mathrm{~cm}$, each for the abathic distance $d_{a}=99.61 \mathrm{~cm}$. The result of these two simulations is shown in Figure 7

We can see that each space between the neighboring iso-disparity lines for disparity $\delta_{0}=0.00355$ shown in Figure 7 s s right panel is filled by an additional five iso-disparity lines for disparity $\delta=0.00071$ shown on the left in Figure 7 . Thus, the relation

$$
\Delta z(z, \delta)=0.000178 z^{2}=\frac{1}{5} 0.000892 z^{2},
$$

can be predicted from the simulations and verified numerically. In fact, substituting $d_{a}=99.61 \mathrm{~cm}$ for $z$ in $(3)$, we obtain, 


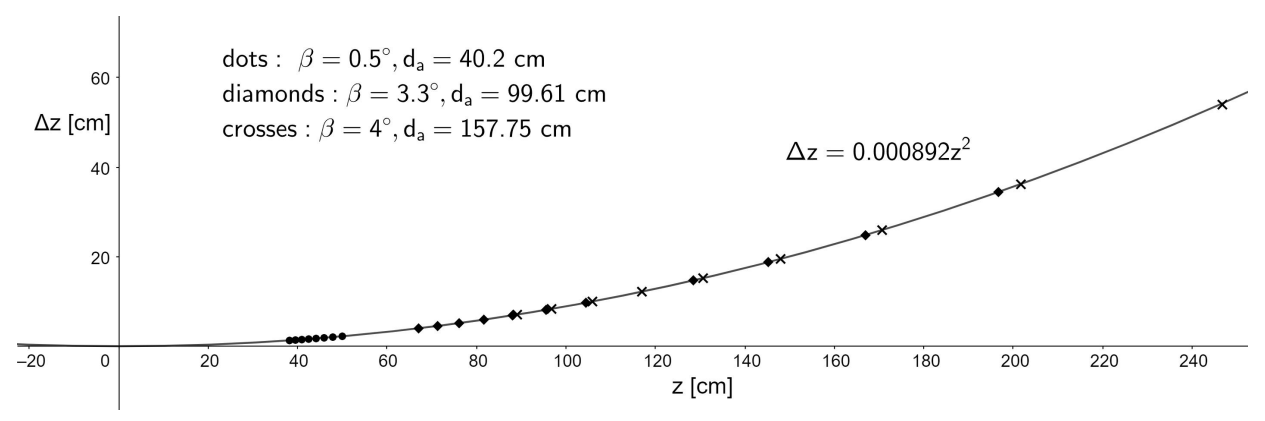

Figure 6: For the simulations shown in Figure 3 the points $(z, \Delta z)$ are plotted for three different abathic distance fixations: $d_{a}=40.2 \mathrm{~cm}$ (dots), $d_{a}=99.61 \mathrm{~cm}$ (diamonds), and $d_{a}=157.75 \mathrm{~cm}$ (crosses).
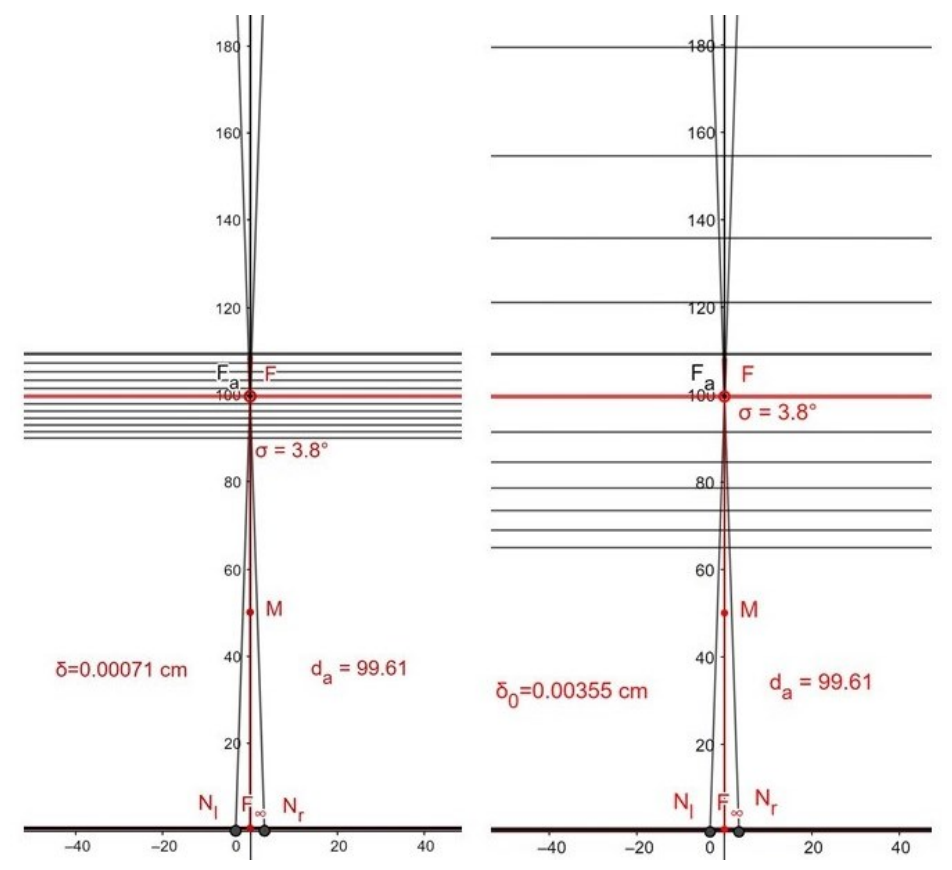

Figure 7: The is-disparity lines for $d_{a}=99.61 \mathrm{~cm}$ with different disparity values; on the right, $\delta_{0}=0.00355 \mathrm{~cm}$, while on the left, $\delta=0.00071 \mathrm{~cm}$.

$$
\begin{aligned}
\Delta z\left(d_{a}, \delta\right) & =0.000178 d_{a}^{2}=1.77 \mathrm{~cm} \\
& =\frac{1}{5} 0.000892 d_{a}^{2}=\frac{1}{5} 8,85 \mathrm{~cm}
\end{aligned}
$$

which agree with the simulations shown in Figure 7

The above discussion of simulations demonstrate that for any $z>0$, disparity $\delta$, and $h>0$,

$$
\Delta z(z, h \delta)=h \Delta z(z, \delta)
$$

This relation can be scaled down to the value of the disparity imposed by the foveal distribution of photoreceptors. I conclude that the interval between iso-disparity lines for the disparity difference $\delta=h \delta_{0}$ satisfies the relation,

$$
\frac{\Delta z\left(z, h \delta_{0}\right)}{z^{2}}=h 0.000892=h \frac{8.85}{(99.61)^{2}}
$$

where $8.85 \mathrm{~cm}$ is the distance between the horopter line and the $-\delta_{0}$-disparity line when $d_{a}=99.61 \mathrm{~cm}$ for $\beta=3.3^{\circ}$. 
I choose the $h$ value such that $\Delta z\left(d_{a}, h \delta_{0}\right)$ gives the depth resolution at the fovea where the cone-to-cone spacing is about $2.7 \times 10^{-4} \mathrm{~cm}$, which gives the corresponding spacing of $10^{-4} \mathrm{~cm}$ on the image plane of the AE. Given that $\delta_{0}=0.00355 \mathrm{~cm}$, we obtain $h=10^{-4} / 3.55 \times 10^{-3} \approx 1 / 35$. Thus, for $h=1 / 35$,

$$
\Delta z\left(d_{a}, h \delta_{0}\right)=h 0.000892 d_{a}^{2}=2.5 \times 10^{-5} d_{a}^{2} .
$$

To simplify notation, I denote $\Delta z\left(z,(1 / 35) \delta_{0}\right)$ by $\Delta z(z)$. Then, we obtain from (7): $\Delta z\left(d_{a}\right) \approx 0.25 \mathrm{~cm}$ for $d_{a}=99.61$ and $\Delta z\left(d_{a}\right) \approx 0.04 \mathrm{~cm}$ for $d_{a}=40.2$.

In order to model relative depth, the simulations must be combined with the fundamental features of the human visual system's architecture, which include the cones' eccentricity dependent density and the convergence of the photoreceptors on the ganglion cells, which is 1-to-1 in the foveala and which approaches 200-to-1 in the periphery. Related to this issue, the concept of receptive fields becomes important. Visual information is sent from the eyes along the ganglion cells' axons and arrives, mainly, at the primary visual cortex, where it is retinotopically mapped with a significant magnification of the foveal region and scaled logarithmically with retinal eccentricity [17].

There is no theory based on first principles that can describe how perceived relative depth varies with disparity and distance. Therefore, using (6), (7) and the above discussion, I postulate that the relative depth element $\delta p$ in visual space approximately satisfies the relation.

Postulate. The relative depth element $\delta d$ in visual space is given by

$$
\delta d=\frac{d_{a}^{2}}{z^{2}} \rho(z) \Delta z(z)=\frac{d_{a}^{2}}{z^{2}} \delta z
$$

where $\delta z=\Delta z\left(d_{a}\right)$. Here $\rho(z)$ accounts for the anatomical and physiological aspects of the human visual system.

Human disparity contains two components, fine disparity and coarse disparity [18]. Fine disparity allows us to determine the depth of objects in the central visual area where disparate images are fused into a single percept (Panum's fusional area). Coarse disparity provides stereopsis from disparities well beyond the fusible range. It is still useful for depth perception for moderately large disparities even for double images but is only clearly signed with a vague impression of depth magnitude for very large disparity values [3, 29]. Importantly, coarse disparity creates our sense of being immersed in the ambient environment [2].

I want to emphasize in this work the modeling of the global aspects of the visual space geometry that can be studied in the Riemannian geometry framework. Some of the global aspects of visual space geometry, which seem to agree with the experimental results reported in the literature, are uncovered by the theory developed here from the relative depth formula postulated in 8 .

\section{Metric Tensor for Resting Eyes}

In this section, I propose a model of the visual space metric tensor, the fundamental concept of Riemannian geometry. To do this, I first derive the expression for the line element in the $y$-direction.

The length of projected $\delta y$ of the iso-disparity line element projected to the Cyclopean eye image is directly proportional to the length of line element $\delta y$ and inversely proportional to the distance to the iso-disparity line. Assuming the veridical line element perception $\delta \lambda$ at the abathic distance,

$$
\delta \lambda(z)=\frac{d_{a}}{z} \delta y
$$

and combining it with (8), we obtain the visual space metric line element

$$
\delta s^{2}=\frac{d_{a}^{2}}{z^{2}} \delta y^{2}+\frac{d_{a}^{4}}{z^{4}} \delta z^{2} .
$$

The binocular field of fixations $B$ in the horizontal plane was discussed in Section 3. This is a convex set parametrized by the coordinates $(y, z)$ that are shown in Figure 2. The set $B$ has the Cyclopean visual metric determined by a map

$$
g:(y, z) \in B \longrightarrow g_{(y, z)} \in M_{2}
$$

where $g_{(y, z)}$ is the metric tensor obtained from 10 , 


$$
g_{(y, z)}=\left(\begin{array}{cc}
\frac{d_{a}^{2}}{z^{2}} & 0 \\
0 & \frac{d_{a}^{4}}{z^{4}}
\end{array}\right),
$$

and $M_{2}$ is the set of all real symmetric positive defined $2 \times 2$ matrices.

The visual metric tensor defines an inner product on each tangent plane $T_{(y, z)} B$, the tangent plane $R^{2}$ at $(y, z)$ that has the origin at this point. Because $(0,0) \notin B$, the map $g$ in $\sqrt{11}$ ) is a smooth function on $B$. The inner product is usually written $\left\langle\partial_{j}, \partial_{k}\right\rangle=g_{p}\left(\partial_{j}, \partial_{k}\right)=g_{j k}(p)$ where $p=(y, z)$ and each index runs over $y$ and $z$ for the vector basis $\partial_{y}, \partial_{z} \in T_{(y, z)} B$. Thus, the length of the vector $V=\left(v^{i}\right)$ is $\|V\|=\sqrt{\langle V, V\rangle}=\sqrt{g_{i j} v^{i} v^{j}}$ and the angle between vectors $U$ and $V$ can be obtained from $\cos \theta=\langle U, V\rangle /\|U\|\|V\|$.

Although I use standard Riemannian geometry notation for writing the basis vectors, it should be understood that for the coordinates $(y, z), \partial_{y}=(1,0)$, and $\partial_{z}=(0,1)$.

The pair $(B, g)$ is a global chart on the binocular field that allows computing length of vectors in tangent spaces and, therefore, length of curves in $B$. It emphasizes global aspects of the visual space.

\section{Geodesic Equations for Resting Eyes}

For an excellent exposition of the Riemannian geometry framework, I refer to [6]. I start by writing the defining expressions for the Christoffel symbols

$$
\Gamma_{j k}^{i}=\sum_{l} \frac{1}{2} g^{i l}\left(g_{l k, j}+g_{j l, k}-g_{j k, l}\right),
$$

where $\left(g^{i l}\right)=\left(g_{i l}\right)^{-1}$ is the inverse matrix.

Then, a geodesic is a curve $\gamma(t)=\left(x^{i}(t)\right)$ whose the covariant derivative of the velocity vector is zero,

$$
\nabla_{t} \frac{d x^{i}(t)}{d t}=\frac{d^{2} x^{i}}{d t^{2}}+\sum_{j} \sum_{k} x_{j k}^{i} \frac{d x^{j}}{d t} \frac{d x^{k}}{d t}=0
$$

In the notation used here, the tensor matrix (12) entries are

$$
g_{y y}=d_{a}^{2} / z^{2} \quad \text { and } \quad g_{z z}=d_{a}^{4} / z^{4}
$$

and the inverse of the tensor matrix 12 entries are

$$
g^{y y}=1 / g_{y y}=z^{2} / d_{a}^{2} \quad \text { and } \quad g^{z z}=1 / g_{z z}=z^{4} / d_{a}^{4}
$$

Adapting this notation used for coordinates $(y, z)$, non-zero expressions 13 are the following:

and

$$
\begin{gathered}
\Gamma_{y z}^{y}=\Gamma_{z y}^{y}=\frac{g_{y y}, z}{2 g_{y y}}=-\frac{1}{z}, \\
\Gamma_{y y}^{z}=-\frac{g_{y y}, z}{2 g_{z z}}=\frac{z}{d_{a}^{2}}
\end{gathered}
$$

$$
\Gamma_{z z}^{z}=\frac{g_{z z}, z}{2 g_{z z}}=-\frac{2}{z} .
$$

Then, using this notation, I obtain the differential equations 14 for the geodesic $\gamma(t)=(y(t), z(t)$ as follows.

$$
y^{\prime \prime}-\frac{2}{z} y^{\prime} z^{\prime}=0
$$


and

$$
z^{\prime \prime}+\frac{z}{d_{a}^{2}} y^{\prime 2}-\frac{2}{z} z^{\prime 2}=0,
$$

where the 'prime' means ' $\frac{d}{d t}$ '. I consider two sets of the initial conditions,

and

$$
y(0)=0, z(0)=z_{1} \text { and } y^{\prime}(0)=k_{1}, z^{\prime}(0)=0
$$

$$
y(0)=y_{1}, z(0)=z_{1} \text { and } y^{\prime}(0)=0, z^{\prime}(0)=k_{2}
$$

To solve this system of equations with the set of initial conditions (22), I first, integrate (20) by separating the variables to obtain $y_{1}^{\prime}=C z^{2}$. By substituting it into 21 and rewriting the equations in terms of $(y(t), w(t))$ where $w=1 / z$, we obtain the system of geodesic equations

$$
y_{1}^{\prime}-\frac{k_{1}}{z_{1}^{2}} w_{1}^{-2}=0
$$

and

satisfying the initial conditions 22,

$$
w_{1}^{\prime \prime}-\frac{k_{1}^{2}}{z_{1}^{4} d_{a}^{2}} w_{1}^{-3}=0
$$

The equation [25] is the simplest of the celebrated Ermakov-Pinney equations [9, 21] distinguished be the fact that the equations has general solutions.

Following [21], for the initial conditions $w_{1}(0)=1 / z_{1}, w_{1}^{\prime}(0)=0$, the solution of $[25]$ satisfying the initial conditions is

$$
w_{1}=\frac{1}{z_{1}}\left(\frac{k_{1}^{2}}{d_{a}^{2}} t^{2}+1\right)^{1 / 2}
$$

For the second set of initial conditions 23 , we have $y_{2}^{\prime}=0$ and, then,

$$
z_{2}^{\prime \prime}=(2 / z) z^{\prime 2}
$$

The geodesics for both initial conditions are derived in the next section where also their basic properties are analysed.

\section{Geodesics in Resting Eyes Visual Space}

For the first set of initial conditions, substituting 26] into 24], we derive the expression for $y^{\prime}$,

$$
y_{1}^{\prime}(t)=k_{1}\left(\frac{k_{1}^{2}}{d_{a}^{2}} t^{2}+1\right)^{-1}
$$

which can be easily integrated,

$$
y_{1}(t)=d_{a} \arctan \left(\frac{k_{1}}{d_{a}} t\right) .
$$

Before I derive the geodesics, I need to calculate $z^{\prime}(t)$. Rewriting 26 as $z(t)=1 / w(t)$ and differentiating it, we obtain,

$$
z_{1}(t)=z_{1}\left(\frac{k_{1}^{2}}{d_{a}^{2}} t^{2}+1\right)^{-1 / 2}
$$




$$
z_{1}^{\prime}(t)=-\frac{z_{1} k_{1}^{2}}{d_{a}^{2}} t\left(\frac{k_{1}^{2}}{d_{a}^{2}} t^{2}+1\right)^{-3 / 2}
$$

For the second set of the initial conditions, integrating [27], we first have

and then we obtain $y_{2}(t)=y_{1}$ and

$$
z_{2}(t)=z_{1}\left(1-\frac{k_{2}}{z_{1}} t\right)^{-1}
$$

$$
z_{2}^{\prime}(t)=\frac{k_{2}}{z_{1}^{2}}\left(1-\frac{k_{2}}{z_{1}} t\right)^{-2}
$$

Theorem. In the resting eyes visual space there are two families of parallel geodesics. The first type of geodesics are starting at a point $\left(0, z_{1}\right) \in B$ in the direction $\partial_{y}$ and the other type of the geodesics are starting at $\left(y_{1}, z_{0}\right)$ in the direction $\partial_{z}$.

Proof. I prove this theorem by showing that the curves satisfying the geodesic equations starting $(1)$ at $\left(0, z_{1}\right)$ in the directions of $\partial_{y}$ and (2) at $\left(0, z_{0}\right)$ in the direction of $\partial_{z}$ have unit speed parametrizations for the corresponding values of $k_{1}$ and $k_{2}$, respectively. For the direction vector $\partial_{y}$, I solve the unit speed condition for the curve $\gamma_{1}(t)=\left(y_{1}(t), z_{1}(t)\right)$

I obtain the unit speed condition

$$
\left\|\gamma_{1}^{\prime}(t)\right\|=\left(g_{y y}\left(z_{1}(t)\right) y_{1}^{\prime 2}+g_{z z}\left(z_{1}(t)\right) z_{1}^{\prime 2}\right)^{1 / 2}=1
$$

$$
k_{1}=\frac{z_{1}}{d_{a}}
$$

Therefore, in this case, the geodesic is given by

$$
\gamma_{1}(t)=\left(d_{a} \arctan \frac{z_{1}}{d_{a}^{2}} t, z_{1}\left(\frac{z_{1}^{2}}{d_{a}^{4}} t^{2}+1\right)^{-1 / 2}\right) .
$$

For the direction vector $\partial_{z}$, the unit speed condition

gives

$$
\left\|\gamma^{\prime}(t)\right\|=\left(g_{z z}\left(z_{2}(t)\right) z_{2}^{\prime 2}\right)^{1 / 2}=1
$$

$$
k_{2}=\frac{z_{1}^{2}}{d_{a}^{2}}
$$

The corresponding geodesic has the expression

$$
\gamma_{2}(t)=\left(y_{1}, z_{1}\left(1-\frac{z_{1}}{d_{a}^{2}} t\right)^{-1}\right)
$$

The graphs of the geodesics for the abathic distances $d_{a}=40.2 \mathrm{~cm}, d_{a}=99.61 \mathrm{~cm}$ and $d_{a}=157.75 \mathrm{~cm}$ shown in 8 ) demonstrate the the geodesics for a given $d_{a}$ value in each of family do not intersect, that is, they are parallel.

The geodesics in the gaze direction $\partial_{z}$, that is, geodesics 39 , can be analysed in details. Solving $z\left(t_{2}\right)=z_{2}$ for $t_{2}$, I derive the distance that does not depends on $y_{1}$,

$$
d\left(z_{1}, z_{2}\right)=t_{2}=d_{a}^{2} \frac{z_{2}-z_{1}}{z_{1} z_{2}}
$$




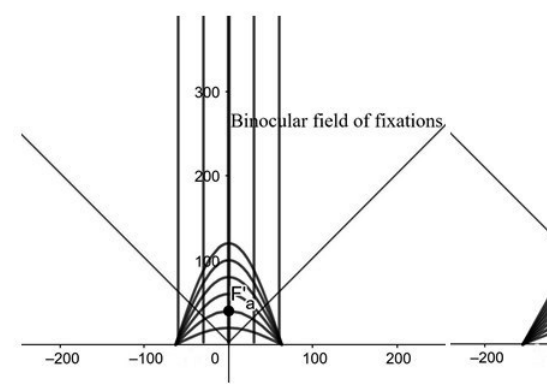

(a)

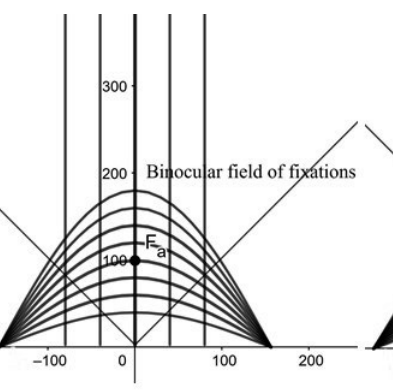

(b)

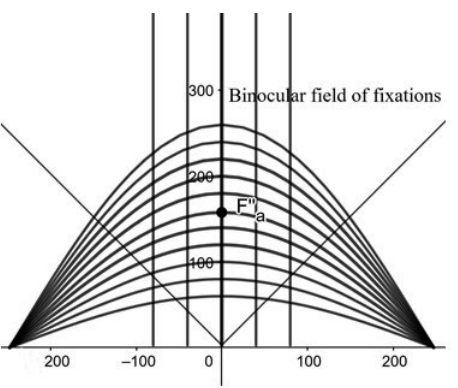

(c)

Figure 8: The geodesics in the resting eyes visual space for (a) $d_{a}=40.2 \mathrm{~cm}$, (b) $d_{a}=99.61 \mathrm{~cm}$, and (c) $d_{a}=157.75$ $\mathrm{cm}$. The curved geodesics start in $\partial_{y}$ direction from the points $\left(0, z_{1}\right)$. They are extended to the whole interval $(-\infty, \infty)$ and approach asymptotically two points on the $y$-axis at $\pm(\pi / 2) d_{a}$. The vertical geodesics start in $\partial_{z}$ direction from the points $\left(y_{1}, z_{0}\right)$. They are defined on the maximal interval $\left(0, d_{a}^{2} / z_{0}\right)$.

The total length of the geodesic from the point $\left(0, z_{0}\right)$, the closest point that can be fixated binocularly, is

$$
d\left(z_{0}, \infty\right)=\lim _{z_{2} \rightarrow \infty} d_{a}^{2} \frac{1-z_{0} / z_{2}}{z_{0}}=\frac{d_{a}^{2}}{z_{0}},
$$

meaning that the $z$-geodesic has the finite length $d_{a}^{2} / z_{0}$ and, therefore, defines the visual horizon. This geodesic is incomplete.

Further, the distance from $\left(0, z_{0}\right)$ to the eyes' resting posture fixation is $d\left(z_{0}, d_{a}\right)=d_{a}^{2} / z_{0}-d_{a}$, which means that the distance from the horopter to the horizon is $d_{a}$.

\subsection{Geodesic Curvature of Iso-disparity Curves}

The iso-disparity curves in physical space are Euclidean lines of constant $z$-values. By the results of the previous section, they are not geodesics. For example, the Euclidean line through $F_{a}$ differs from the geodesic line passing through the same point that is shown in (8). How far this iso-disparity line differs from the geodesic is given by its geodesic curvature since every geodesic has zero geodesic curvature, see [15], page 137.

The unit speed iso-disparity curve is $\left.\left(y(t), z_{1}\right)=\left(z_{1} / d_{a}\right) t, z_{1}\right)$. It has the acceleration components

and

$$
\nabla_{t} y^{\prime}(t)=\Gamma_{y y}^{y}\left(z_{1}\right) y^{\prime}(t) y^{\prime}(t)=0
$$

$$
\nabla_{t} z^{\prime}(t)=\Gamma_{y y}^{z}\left(z_{1}\right) y^{\prime}(t) y^{\prime}(t)=\frac{z_{1}^{3}}{d_{a}^{4}} .
$$

The geodesic curvature of the iso-disparity curve is

$$
\kappa=\left\|\left(\nabla_{t} y^{\prime}(t), \nabla_{t} z^{\prime}(t)\right)\right\|=\sqrt{\frac{d_{a}^{4}}{z_{1}^{4}} \frac{z_{1}^{6}}{d_{a}^{8}}}=\frac{z_{1}}{d_{a}^{2}} .
$$

Thus, the iso-disparity line (a Euclidean line) for the resting eyes at the abathic distance $d_{a}$ that is passing through $\left(0, z_{1}\right)$ is a curve with a constant geodesic curvature $z_{1} / d_{a}^{2}$. In particular, the horopteric line, in this case, has the geodesic curvature $1 / d_{a}$.

\section{The Riemann Curvature}

Here the derivation of the Riemann curvature of visual space is only sketched for the eyes' resting vergence posture and the reader is referred to [15] a complete introduction to this complicated object. In the basis vectors $\partial_{i}$, where the index runs here over $y$ and $z$, the covariant differentiation $\nabla_{\partial_{i}}$ on the basis vectors is 


$$
\nabla_{\partial_{j}} \partial_{k}=\sum_{m} \Gamma_{j k}^{m} \partial_{m}
$$

Following [15], the index notation of the curvature tensor can be written as,

$$
\sum_{m} R_{j k l}^{m} \partial_{m}=\nabla_{\partial_{j}} \nabla_{\partial_{l}} \partial_{k}-\nabla_{\partial l} \nabla_{\partial_{k}} \partial_{j},
$$

which, in low indices, is the following:

$$
R_{i j k l}=\sum_{m} g_{i m} R_{j k l}^{m} .
$$

For two-dimensional Riemannian manifolds, $R_{1212}$ yields all components of the Riemann curvature tensor up to a sign. Thus, using

$$
R_{1212}=(1 / 2)\left(g_{12,21}+g_{21,12}-g_{11,22}-g_{22,11}\right)+g_{p q}\left(\Gamma_{12}^{p} \Gamma_{21}^{q}-\Gamma_{11}^{p} \Gamma_{22}^{q}\right)
$$

and by making the use of (13), (17), (18), (19), 45), (46) and (47), I derive in the notation corresponding to the coordinates $(y, z)$,

$$
\begin{aligned}
R_{y z y z} & =-\frac{1}{2} g_{y y, z z}+\frac{g_{y y, z}^{2}}{4 g_{y y}}+\frac{g_{y y, z} g_{z z, z}}{4 g_{z z}} \\
& =-\frac{3 d_{a}^{2}}{z^{4}}+\frac{d_{a}^{2}}{z^{4}}+\frac{2 d_{a}^{2}}{z^{4}}=0
\end{aligned}
$$

Thus, in this case of the eyes' resting vergence posture, the curvature tensor of visual space vanishes identically and visual space is flat for this particular eye fixation [15].

\section{Discussion}

We note first, as the simulations clearly show, that visual space geometry is shaped by physical space being expanded between the head and the frontal line horopter at the abathic distance $d_{a}$ and compressed beyond the horopter. These changes in perceived depth when the eyes are in their resting vergence posture (corresponding to the eye's lens tilt's angle $\beta$ ) make the geodesics incomplete such that visual space is bounded by the horizon in the direction of the gaze.

For the numerical calculations, I assume the human average lens tilt $\beta=3.3^{\circ}$, which gives the average abathic distance $d_{a}=99.61 \mathrm{~cm}$ [26]. I also estimate that $z_{0}=3 \mathrm{~cm}$. Using these values in the geodesic distance expressions, I obtain the total distance to the horizon,

$$
d\left(z_{0}, \infty\right)=\frac{d_{a}^{2}}{z_{0}}=\frac{0.9961^{2}}{0.03} \approx 33 \mathrm{~m},
$$

and the distance to the horopter at the abathic distance fixation,

$$
d\left(z_{0}, d_{a}\right)=\frac{d_{a}^{2}}{z_{0}}-d_{a} \approx 32 \mathrm{~m} .
$$

Therefore, the distance from the horopter at the abathic distance to the horizon is $d\left(d_{a}, \infty\right) \approx 1 \mathrm{~m}$.

The finite distance to the horizon should be expected as we perceive objects in the horizontal binocular field against the horizon with the depth perception resulting from the metric 10 such that our field of vision appears two-dimensional with the objects initially located in visual processing at different distances from the horopter and finally perceived against the egocentric coordinates.

In literature, the distance of the vanishing points at the horizon is reported to be between $6 \mathrm{~m}$ and $100 \mathrm{~m}$, see [8]. Here, the AE model in the binocular system determines the eyes' resting vergence posture abathic distance that depends on the lens tilt angle $\beta$. The simulations were carried out for three values of the abathic distances; $d_{a}\left(0.5^{\circ}\right)=40.2$ 
bioRxiv preprint doi: https://doi.org/10.1101/2021.10.25.465820; this version posted January 11, 2022. The copyright holder for this preprint (which was not certified by peer review) is the author/funder. All rights reserved. No reuse allowed without permission.

A PREPRINT - JANUARY 11, 2022

$\mathrm{cm}, d_{a}\left(3.3^{\circ}\right)=99.61 . \mathrm{cm}$, and $d_{a}\left(4^{\circ}\right)=157.75 \mathrm{~cm}$. For these values, I obtain the distance to the horizon $5.4 \mathrm{~m}, 33$ $\mathrm{m}$, and $83 \mathrm{~m}$, respectively. Thus, the geometric theory of the binocular perception developed in [26] and extended here to iso-disparity curves, unifies in one theory the distances to the horizon reported in the literature, where precise explanations for the differences in these distances were not provided.

The curvature of visual space, derived here for the eyes' resting vergence posture when they fixate at the abathic distance, is zero, so the visual space is flat for this particular eyes posture. In this case, the iso-disparity curves in physical space are $z$-coordinates. However, the iso-disparity curve through $\left(0, z_{1}\right)$ for fixation at the abathic distance $d_{a}$ has a non-zero geodesic curvatures $\kappa=z_{1} / d_{a}^{2}$. The geodesics starting at $\left(0, z_{1}\right)$ in direction $\partial_{y}$ consists families for each of abathic distances $d_{a}$ such that each family asymptotically converges to two points $\pm\left((\pi / 2) d_{a}, 0\right)$ on the $y$-axis, see Figure (8). Therefore, they are parallel.

For all other fixations, the iso-disparity conics consist of the two families of iso-disparity curves in Figure 4 and Figure 5. for short-distance fixations the iso-disparity curves are ellipses, while for far-distance fixations the iso-disparity curves are hyperbolas. For the precise classification of the horopter's shape and, hence, the iso-disparity curves' shape with respect to the fixation point in the binocular field, see [26].

Because the convergence in the case of the elliptic iso-disparity family, the metric tensor will depends on the $y$ coordinates and the curvature of visual space should be positive. Similarly, because of the divergence in the case of the hyperbolic iso-disparity family, the curvature of visual space should be negative. The numerical value of the curvature cannot be readily obtained without the known metric tensor [5].

The results obtained here, although not complete, revise the abstract geometric theories of binocular phenomenal space, most notably, a constant negative-curvature hyperbolic geometry of visual space originally proposed by Luneburg [16] in the 1940s and further developed in [4] and [12], among other publications.

Luneburg's approach is one of three theories that used axiom-based model geometries: Euclidean, hyperbolic, and spherical, to model phenomenal spatial relations [28]. These approaches, however, were not supported by experimental data and did not pay attention to biological and psychological aspects of human vision. For example, Luneburg's theory uses the Vieth-Müller circle (VMC) as the geometric horopter. In [24], I showed that the VMC is anatomically inaccurate as a geometric horopter. In fact, it incorrectly assumes the nodal point located at the eye's rotation center [11], which provides a good numerical approximation of the geometric horopter close to the fixation point. More significantly, when the VMC is compared with the simulated horopteric hyperbolas in [26], their difference in periphery is very large. Thus, the modeling based on the VMC will significantly distort the ambient space geometry provided by the coarse disparity, see the discussion in Section 5 .

Beyond these three theories, there are also theories based on Riemann's approach to studying abstract space with defined metric function. One of the most general distance-defined spaces is called metric space. These theories model visual space's measurable properties such as distances and angles while also including stimulus location in physical space and psychological factors [28]. The theory proposed here for modeling visual space spatial relations is similar to the metric-based theories but also includes anatomical and physiological aspects of the human visual system.
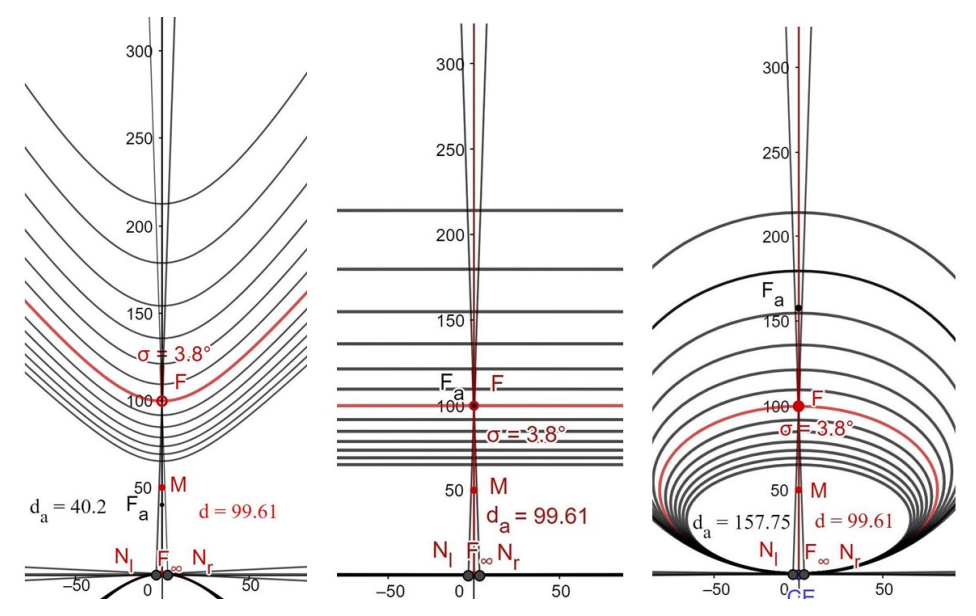

Figure 9: Three simulations for the same fixation and different values of the AE's lens' tilt. On the left, the lens' tilt $\beta=0.5^{\circ}$, in the middle, $\beta=3,3^{\circ}$, and on the right, $\beta=4^{\circ}$. In these cases, the curvature should be negative, zero and positive, respectively. 
Of the many other studies reporting results on phenomenal geometry, the experimentally obtained values of the visual space's curvature in [14] could be closest to the results obtained in the presented here studies.

[14] found that the curvature changes from elliptic in near space to hyperbolic in far space. For very large distances, the visual plane became parabolic. Although, the values of the curvature can only be conjecture from Figure 9 in general it support the experimental results in [14].

I conclude this section by emphasizing that the geometry of visual space, when eyes are in the resting vergence posture, that is, when eyes fixate at the abathic distance, is determined by the choice of the metric for this particular eyes posture. This geometry asserts that the iso-disparity frontal lines determine the parallel family of geodesics around the gaze direction in physical space, although incomplete. This incompleteness determines the finite distance to the horizon for each abathic distance to the fixation point. Further, the iso-disparity frontal lines themselves have nonzero geodesic curvature, meaning that they are not geodesics. Nevertheless, this visual space has zero curvature.

For other than the resting eyes posture fixations, the families of iso-disparity curves either contain elliptic or hyperbolic curves. In the above discussion, I assumed that the geodesics around the gaze direction, in these cases, are curved in physical space. The result of the iso-disparity shapes is that the visual space curvatures have nonzero values: positive for elliptic iso-disparity curves and negative for hyperbolic disparity curves.

\section{Acknowledgements}

I thank Alice Turski for her help with editing this manuscript.

\section{References}

[1] P. Artal, Optics of the eyes and its impact in vision, Adv. Opt. Photon, 6, 340-367 (2014).

[2] S.R. Barry, Beyond the critical period. Acquiring stereopsis in adulthood. In Plasticity in Sensory Systems. Steeves J.K. and Harris L.R. (eds.). Cambridge University Press. 175-195 (2013).

[3] C. Blakemore, The Range and Scope of Binocular Depth Discrimination in Man. J. Physiol. 211, 599-622 (1970).

[4] A.A. Blank, The Luneburg theory of binocular visual space, J. Opt. Soc. Am., 43, 717-727 (1953).

[5] K. Burns, V.S. Matveev, Open problems and questions about geodesics, Ergodic Theory and Dynamical Systems, 41, 641-684 (2021).

[6] M.P. Do Carmo, Riemannian Geometry, Birkhäuser, Boston, 1993.

[7] S.M. Ebenholtz, Oculomotor Systems and Perception, Cambridge U Press, 2001.

[8] C.J. Erkelens, Perspective space as a model for distance and size perception, i-Perception, 1-20 (2017).

[9] P. Ermakov, Second Order Differential Equations. Conditions of Complete Integrability, Universita Izvestia Kiev 9, 1-25 (1880). (Russian).

[10] D. Guitton, M. Volle, Gaze control in humans: eye-head coordination during orienting movements to targets within and beyond the oculomotor range, J. Neurophysiol., 58, 427-459 (1987).

[11] W.L. Gulick and R.B. Lawson, Human stereopsis: A psychophysical analysis. New York: Oxford University Press 1976.

[12] T. Indow, A critical review of Luneburg's Model with regard to global structure of visual space, Psychological Review, 98, 430-453 (1991).

[13] W. Jaschinski-Kruza, Eyestrain in VDU users: viewing distance and the resting position of ocular muscles, Hum. Factors, 33, 69-83 (1991).

[14] J.J. Koenderink, A.J. van Doom, J. S. Lappin, Direct measurement of the curvature of visual space, Perception, 29, 69-79 (2000).

[15] J.M. Lee, Riemannian Manifolds: An Introducton to Curvature, Springer. 1997.

[16] R.K. Luneburg, Mathematical Analysis of Binocular Vision, Princeton University Press, 1947.

[17] G. Maiello, M. Chessa, P.J. Bex, F. Solari, Near optimal combination of disparity across a log-polar scaled visual field, PLoS Comp. Biol., 16(4):e1007699 (2020).

[18] A.M. Norcia, E.E. Sutter, C.W. Tyler, Electrophysiological evidence for the existence of coarse and fine disparity mechanisms in human, Vis. Res., 25, 1603-1611 (1985). 
[19] K. N. Ogle, Precision and validity of stereoscopic depth perception from double image, J. Opt. Soc. Am. 41, 906-913 (1953).

[20] K. Ogle, Researches in Binocular Vision, PA:WB Saunders, Philadelphia, 1950.

[21] E. Pinney, The nonlinear differential equation $y^{\prime \prime}+p(x) y+c y^{-3}$, Proc. Am. Math. Sc. 1, 681 (1950).

[22] T. Shipley, S. Rawlings, The Nonius Horopter-I. History and Theory, Vis. Res. 10. 1225-1262 (1970).

[23] T. Shipley and S. Rawlings, The Nonius Horopter-II. An Experimental Report, Vis. Res., 10, 1263-1299 (1970).

[24] J. Turski, On binocular vision: the geometric horopter and cyclopean eye, Vis. Res., 119, 73-81 (2016).

[25] J. Turski, Binocular system with asymmetric eyes, J. Opt. Soc. Am. A 35, 1180-1191 (2018).

[26] J. Turski, A geometric theory integrating human binocular vision with eye movement, Front. Neurosci. 14:555965, 1-17 (2020).

[27] G. von Noorden and E.C. Campos, Binocular Vision and Ocular Motility: Theory and Management of Strabismus. Mosby, A Harcourt Health Sciences Company, St. Louis, 2002.

[28] M. Wagner, The Geometries of Visual Space, Lawrence Erlbaum Associates, Mahwah, NJ, 2006.

[29] L.M. Wilcox and R.S. Allison, Coarse-fine dichotomies in human stereopsis. Vis. Res. 49,2653-2665 (2009). 